11

\title{
Влияние межатомного обменного взаимодействия на магнитные фазовые переходы в условиях спинового кроссовера при высоких давлениях
}

\author{
(C) С.Г. Овчинников ${ }^{1,2}$, Ю.С. Орлов ${ }^{1,2, \uparrow}$, С.В. Николаев ${ }^{1,2}$, А.И. Нестеров ${ }^{3}$, Т.М. Овчинникова ${ }^{4}$ \\ ${ }^{1}$ Сибирский фредеральный университет, \\ Красноярск, Россия \\ ${ }^{2}$ Институт физики им. Л.В. Киренского, ФИЦ КНЦ СО РАН, \\ Красноярск, Россия \\ ${ }^{3}$ Departamento de Fisica, Universidad de Guadalajara, \\ Guadalajara, Mexico \\ ${ }^{4}$ Инситут леса им. Л.В. Сухачева, ФИЦ КНЦ СО РАН, \\ Красноярск, Россия \\ ฯ E-mail: jso.krasn@mail.ru
}

Рассчитана фазовая диаграмма антиферромагнетика со спиновым кроссовером из высокоспинового состояния в низкоспиновое $S=0$ с ростом внешнего давления с учетом зависимости обменного интеграла от давления. Результаты расчета сравниваются с экспериментальными данными по ферропериклазу $\mathrm{Fe}_{x} \mathrm{Mg}_{1-x} \mathrm{O}$.

Работа выполнена при финансовой поддержке Российского фонда фундаментальных исследований, Правительства Красноярского края, Красноярского краевого фонда поддержки научной и научно-технической деятельности в рамках научных проектов № 16-42-243048, 16-42-240413, Совета по грантам Президента РФ (СП-1844.2016.1), Российского фонда фундаментальных исследований (гранты 16-02-00507, 16-02-00273, 18-02-00453).

DOI: 10.21883/FTT.2018.06.45994.01M

\section{1. Введение}

Спиновые кроссоверы в магнитных диэлектриках наблюдаются в большом числе оксидов железа в экспериментах с алмазными наковальнями, типичное значение критического давления $P_{c}=50-70 \mathrm{GPa}$ [1]. Обычно магнитное состояние иона железа регистрируется по эффекту Мессбауэра. Изменение спинового состояния между высокоспиновым (HS) и низкоспиновым (LS) можно также увидеть в спектрах рентгеновского излучения, что особенно удобно для образцов в парамагнитной фазе. Кроме того, зависимость объема вещества от давления чувствительна к типу спинового состояния, поскольку ионный радиус LS-иона значительно (около 10\%) меньше, чем HS-иона. Традиционно теоретическое описание спиновых кроссоверов основано на одноионной картине, в которой HS-состояние стабилизируется внутриатомным хундовским обменным интегралом, a LS-состояние стабилизируется кристаллическим полем, которое растет с ростом давления. Поэтому типичное для изолированного атома HS-состояние сменяется LS-состоянием, когда энергия кристаллического поля сравнивается с хундовским обменом. В рамках такой картины спиновый кроссовер при нулевой температуре является квантовым фазовым переходом по давлению с топологическим параметром порядка [2], но при конечной температуре фазового перехода нет и спиновый кроссовер заключается в плавном переходе от одного к другому состоянию. Вопрос, является ли спиновый кроссовер термодинамическим фазовым переходом или нет, остается открытым.
Экспериментальные исследования спиновых кроссоверов в большом классе магнитных оксидов показывают в ряде случаев скачок объема при кроссовере, но не всегда [1].

В настоящей работе мы анализируем влияние межатомного обменного взаимодействия на спиновый кроссовер. Нами исследована модель с LS-термом $S=0$, что соответствует $d^{6}$-соединениям, например, ферропериклазу $\mathrm{Fe}_{x} \mathrm{Mg}_{1-x} \mathrm{O}$. В приближении среднего поля показано, что на магнитной фазовой диаграмме на плоскости $(T, P)$ при учете межатомного обмена появляется линия фазовых переходов первого рода по давлению из антиферромагнитного HS-состояния в немагнитное LSсостояние в окрестности критического давления, которое само зависит от температуры [3]. Такое поведение характерно для температур ниже трикритической точки $T^{*}<T_{\mathrm{N}}$. Выше трикритической точки переход по давлению будет переходом второго рода. Выше точки Нееля спиновый кроссовер, как и в отсутствие межатомного обмена, заключается в плавном переходе от одного к другому состоянию. Температура Нееля при малых давлениях линейно растет в связи с ростом межатомного обменного взаимодействия.

\section{2. Эффективный гамильтониан в приближении среднего поля}

В соединениях со спиновым кроссовером состояние системы задается спиновыми и орбитальными кван- 
товыми числами. Вблизи спинового кроссовера существенны только два состояния (HS и LS), которые мы можем различать псевдоспином $\tau_{z}=+1 / 2$ и $\tau_{z}=-1 / 2$. Микроскопический вывод эффективного гамильтониана, описывающего возможность спинового кроссовера и магнитного порядка за счет межатомного обмена (упорядочения по спину), был недавно сделан нами в рамках многоэлектронного LDA + GTB-метода [4].

В случае $d^{6}$-ионов, для которых $S_{\mathrm{HS}}=2$ и $S_{\mathrm{LS}}=0 \mathrm{~S}$. Эффективный гамильтониан [4] в приближении среднего поля для спиновых и псевдоспиновых переменных в антиферромагнитной фазе имеет вид

$$
H=H_{0}-\sum_{i} \mathbf{B S}_{i}-\Delta_{\mathrm{eff}} \sum_{i} \tau_{i}^{z} .
$$

Здесь $\mathbf{B}=z J S n^{2}\langle\mathbf{m}\rangle$ - двухподрешеточное среднее поле. $\mathbf{S}_{i}$ и $\tau_{i}^{z}$ - операторы спина и псевдоспина на узле кристаллической решетки $i, \tau_{i}^{z}|\alpha\rangle=\lambda_{\alpha}|\alpha\rangle$. Индекс $\alpha$ принимает значения 1 и 2, соответствующие HS- и LS-состояниям соответственно. Собственные значения $\lambda_{\alpha}$ равны: $\lambda_{1}=1$ и $\lambda_{2}=-1 .\langle\mathbf{m}\rangle=(0,0, m)-$ среднее значение вектора намагниченности $\left\langle\mathbf{S}_{i}\right\rangle=S\left\langle\mathbf{m}_{i}\right\rangle$

$$
\begin{gathered}
H_{0}=\frac{N v m^{2}}{2} n(3 n-1)-\frac{N v}{2} n(1-n), \\
\Delta_{\mathrm{eff}}=\frac{v}{2}\left(1+m^{2}\right) n+\varepsilon_{0}-f(P),
\end{gathered}
$$

где $v=z J S^{2}, z-$ число ближайших соседей, $g=g_{\mathrm{HS}} / g_{\mathrm{LS}}-$ отношение кратностей вырождения HS- и LS-состояний $\left(g=15\right.$ для ионов $\left.\mathrm{Fe}^{2+}\right), \beta-$ обратная температура, $n$ - заселенность HS-состояния, $N$ - число узлов решетки. $\varepsilon_{0}=\Delta_{S} / 2$, где $\Delta_{S}=E_{\mathrm{LS}}-E_{\mathrm{HS}}-$ величина спиновой щели (энергетический интервал между LS- и HS-состояниями) при нулевом давлении. В дальнейшем мы будем предполагать линейную зависимость изменения кристаллического поля от давления: $f(P)=a P$, точка кроссовера $P=P_{C}$ определяется условием $\varepsilon_{0}=f\left(P_{C}\right)$, и линейную зависимость обменного интеграла $J$ от давления: $J(P)=J_{0}+b P$ [5]. Уравнения самосогласования для намагниченности подрешетки $m$ и чисел заполнения $n$, определяющих среднее значение псевдоспина, имеют следующий вид:

$$
\begin{gathered}
m=B_{S}\left(\beta v m n^{2}\right), \\
n=\frac{1+\tanh \left(\beta \Delta_{\mathrm{eff}}(P)+\ln \sqrt{g}\right)}{2},
\end{gathered}
$$

где $B_{S}(x)$ - функция Бриллюэна.

\section{3. $P-T$-фазовая диаграмма}

Рассмотрим сначала решения системы уравнений (4) и (5) в отсутствии обменного взаимодействия, при $J=0$. В этом случае будем иметь $m=0$ для намагниченности

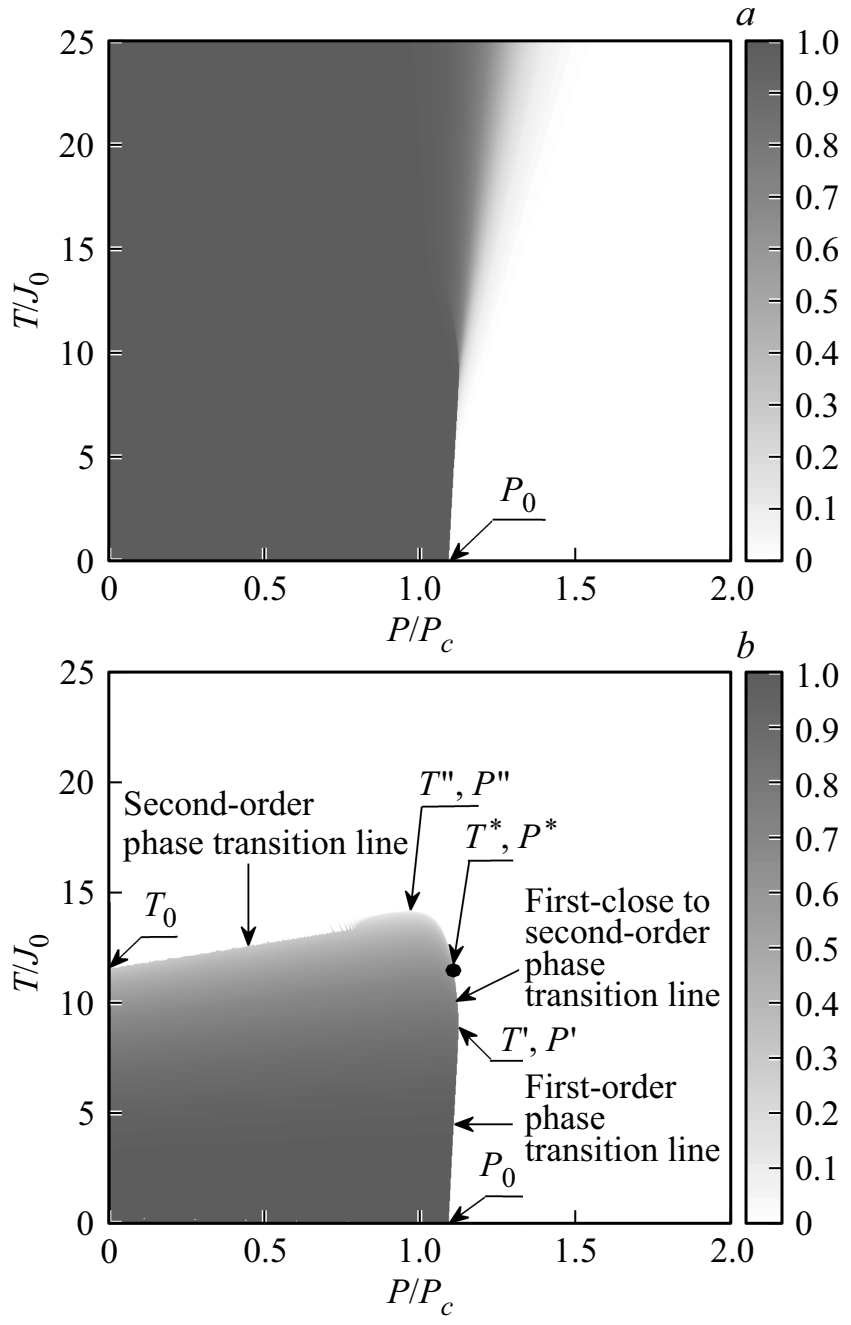

Диаграмма заселенности HS-состояния $n(a)$ и намагниченности $m(b)$, соответствующая минимуму свободной энергии $F$.

и резкий скачок заселенности HS-состояния в точке кроссовера при $T=0$, соответствующий квантовому фазовому переходу [2]. При $J=0$ квантовый фазовый переход с ростом температуры размывается в плавный кроссовер. При учете обменного взаимодействия уравнения (4), (5) решались численно. Для заданных значений температуры и давления возможно появление нескольких решений для параметров $m$ и $n$, из которых мы выбираем решения, соответствующие минимуму свободной энергии Гельмгольца

$$
F=H_{0} / N-k_{\mathrm{B}} T \ln Z_{S}-k_{\mathrm{B}} T \ln Z_{\tau},
$$

где

$$
Z_{S}=\frac{\sinh \left(\left(1+\frac{1}{2 S}\right) \beta v m n^{2}\right)}{\sinh \left(\frac{1}{2 S} \beta v m n^{2}\right)}
$$

и

$$
Z_{\tau}=2 \sqrt{g} \cosh \left(\beta \Delta_{\mathrm{eff}}+\ln \sqrt{g}\right) .
$$

Внешнее давление и температура приведены в единицах $P_{C}$ и обменного взаимодействия $J_{0}$ соответственно. 
Здесь и ниже расчеты были выполнены для следующих значений набора параметров: $J_{0}=28 \mathrm{~K}, S=2$, $z=6, \quad g=15, \quad a=80 \mathrm{~K} \cdot \mathrm{GPa}^{-1}, \quad b=0.5 \mathrm{~K} \cdot \mathrm{GPa}^{-1}$, $P_{C}=55 \mathrm{GPa}$.

На рисунке в координатах давления и температуры представлены диаграммы заселенности HS-состояния $n(a)$ и намагниченности $m(b)$, являющихся самосогласованным решением системы уравнений (4) и (5). Видно, что из-за наличия кооперативного обменного взаимодействия $J$ в системе сохраняется основное магнитоупорядоченное состояние вплоть до $P_{0}>P_{C}$, несмотря на то, что в одноионной картине при $P>P_{C}$ основным является немагнитное LS-состояние. При $P>P_{0}$ основное магнитное состояние сменяется немагнитным путем перехода первого рода.

В области давлений $P \leq P_{0}$ (рисунок, $b$ ) с ростом температуры система испытывает фазовый переход второго рода в парамагнитное состояние. На $P-T$-диаграммах хорошо видно существование особой точки, т.н. трикритической точки $\left(T^{*}\right.$ и $P^{*}$ на рисунке, $\left.b\right)$, в которой линия фазовых переходов второго рода непрерывно переходит в линию фазовых переходов первого рода. При $P_{0}<P \leq P^{\prime}$ основное состояние системы является не магнитным, но с ростом температуры заселяется магнитное HS-состояние, и в системе путем фазового перехода первого рода восстанавливается дальний магнитный порядок (рисунок, $b$ ), как энергетически более выгодный. Таким образом, благодаря кооперативному взаимодействию $J$ в системах со спиновым кроссовером под давлением возможно существование возвратной намагниченности. При дальнейшем увеличении температуры система переходит в парамагнитное состояние путем фазового перехода второго рода, если $P_{0}<P \leq P^{*}$, и первого рода, близкого ко второму, если $P^{*}<P \leq P^{\prime}$. С ростом давления при $P>P_{C}$ увеличивается энергетический интервал между основным немагнитным LS- и ближайшим возбужденным магнитным HS-состоянием и при $P>P^{\prime}$ (рисунок, $b$ ) тепловая энергия, необходимая для заселения HS-состояния в нужной степени, становится сопоставимой с величиной обменного взаимодействия $J-$ магнитный порядок не возникает.

Кроме существования возвратной намагниченности по температуре при $P_{0}<P \leq P^{\prime}$, с учетом увеличения обменного интеграла с ростом давления становится возможным существование возвратной намагниченности по давлению при $T_{0}<T \leq T^{\prime \prime}$, где $T_{0}-$ температура Нееля при $P=0$, а $T^{\prime \prime}-$ максимально возможное значение температуры Нееля с ростом давления. Так при $T_{0}<T \leq T^{\prime \prime}$ (рисунок, $b$ ) система из парамагнитного состояния с ростом давления сначала переходит в магнитоупорядоченное состояние путем фазового перехода второго рода, а потом в немагнитное путем фазового перехода второго рода, если $T^{*}<T_{0}$ и либо первого рода, если $T^{*}>T_{0}$ и $T_{0}<T<T^{*}$, либо второго рода, если $T^{*}>T_{0}$, но $T^{*}<T<T^{\prime \prime}$. В нашем случае для используемого набора параметров $T^{*}<T_{0}$.

\section{4. Заключение}

Обменное взаимодействие вблизи спинового кроссовера под давлением приводит к необычному перестроению системы. Магнитное упорядочение может быть подавлено внешним давлением, а квантовый фазовый переход с ростом температуры перестраивается сначала в переход первого, а потом второго рода. Благодаря кооперативному межатомному обменному взаимодействию в системах со спиновым кроссовером под давлением возможно существование возвратной намагниченности по температуре и по давлению.

\section{Список литературы}

[1] И.С. Любутин, А.Г. Гаврилюк. УФН 179, 1048 (2009).

[2] A.I. Nesterov, S.G. Ovchinnikov. Pis'ma v ZhETF 90, 580 (2009).

[3] Ю.С. Орлов, С.В. Николаев, А.И. Нестеров, С.Г. Овчинников. Письма в ЖЭТФ 105, 732 (2017).

[4] A.I. Nesterov, Yu.S. Orlov, S.V. Nikolaev, S.G. Ovchinnikov. Phys. Rev. B печати.

[5] I.S. Lyubutin, S.G. Ovchinnikov. JMMM 324, 3538 (2012). 